\title{
Longevidade pós-colheita de flores de Oncidium varicosum (Orchidaceae)
}

\section{Post-harvest longevity of Oncidium varicosum (Orchidaceae) flowers}

\author{
Fábio Alcovér Hastenreiter ${ }^{1}$; José Geraldo Zaparoli Vieira ${ }^{2}$; Ricardo Tadeu Faria ${ }^{3 *}$
}

Resumo

\begin{abstract}
A orquídea Oncidium varicosum é bastante utilizada como flor de vaso e atualmente vem se destacando como flor de corte. Devido sua baixa resistência pós-colheita, o objetivo deste trabalho foi avaliar a longevidade de Oncidium varicosum após o acondicionamento em soluções conservantes com diferentes concentrações de sacarose. As hastes de Oncidium varicosum, produzidas em Holambra (SP), foram transportadas para Londrina (PR) em caminhão climatizado a $5-7^{\circ} \mathrm{C}$. Foram avaliadas a utilização de prétratamento com "pulsing" ( $15 \%$ de sacarose por 24 horas) seguido de acondicionamento em solução de manutenção (1, 2, 3 e 4\% de sacarose) e o acondicionamento em solução de manutenção sem prétratamento. As hastes florais foram distribuídas em delineamento inteiramente casualizado, com dez tratamentos e cinco repetições. As avaliações das flores foram diárias, sob critério de notas definido para comparar e verificar a conservação dos aspectos visuais. Nenhum dos tratamentos demonstrou superioridade à testemunha na manutenção da longevidade das flores, que foi de três a quatro dias.
\end{abstract}

Palavras-chave: "Pulsing", sacarose, flor de corte

\begin{abstract}
Oncidium varicosum from the Orchidaceae family has been widely used as pot flower, and, most recently, as cut flower. The objective of this work was to evaluate the longevity of the Oncidium varicosum, due to its low post-harvest resistance, after being conditioned in preservation solutions with different concentrations of sucrose. Oncidium varicosum stems grown in Holambra (SP) were transported to Londrina (PR) in climatized trucks at $5-7^{\circ} \mathrm{C}$. Pre-treatment with pulsing ( $15 \%$ sucrose for 24 hours) followed by conditioning in maintenance solution $(1,2,3$, and $4 \%$ of sucrose) as well as conditioning in maintenance solution without pre-treatment were evaluated. Flower stems were distributed in an entirely casualized design, with ten treatments and five replications. Flowers were evaluated daily, using a score system developed to compare and verify the conservation of visual aspects. No treatment was superior to the control in maintaining the longevity of the flowers.
\end{abstract}

Key words: "Pulsing", sucrose, cut flowers

1 Engenheiro Agrônomo formado Universidade Estadual de Londrina, Londrina-Pr. E- mail: fabioalcover@hotmail.com.br

2 Engenheiro Agrônomo Aluno regular do curso de Pós-graduação em Agronomia, Área de concentração em Fitotecnia, Departamento de Agronomia Universidade Estadual de Londrina, Londrina-Pr. E-mail: zapavieira@terra.com.br

3 Engenheiro Agrônomo Aluno regular do curso de Pós-graduação em Agronomia, Área de concentração em Fitotecnia, Departamento de Agronomia Universidade Estadual de Londrina, Londrina-Pr. E-mail: zapavieira@terra.com.br

* Autor para correspondência. 


\section{Introdução}

A orquídea Oncidium varicosum é bastante utilizada como flor de vaso e atualmente vem se destacando como uma das tendências como flor de corte. Entretanto, não foram encontradas referências à utilização de tratamentos pós-colheita visando prolongar sua conservação.

Visando elevar a qualidade da produção nacional de flores e plantas ornamentais e atender às exigências do mercado internacional, foi implantado em janeiro de 2001, por meio de um convênio entre o Instituto Brasileiro de Floricultura (IBRAFLOR) e a Agência de Promoção de Exportação (APEX), o Programa FloraBrasilis. Segundo o Instituto Brasileiro de Floricultura (2005) o aumento dos investimentos realizados na Cadeia Produtiva de Flores e Plantas Ornamentais, com vistas à melhoria da qualidade e competitividade das exportações brasileiras, tornouse uma realidade visível nos resultados, cada vez maiores e mais dinâmicos, das vendas internacionais.

Os valores exportados no ano de 2004 somaram aproximadamente US\$23,5 milhões, acrescentado mais de $21 \%$ sobre o total de vendas do mesmo período de 2003 e quase $40 \%$ sobre a média dos últimos quatro anos. Somente as flores de corte foram responsáveis por aproximadamente US\$ 4,9 milhões das exportações, (INSTITUTO BRASILEIRO DE FLORICULTURA, 2005).

Ainda segundo o Instituto Brasileiro de Floricultura (2005) o setor é responsável pela geração de em torno de 50 mil empregos. Desses, 22,5 mil trabalhadores estão na produção, 3,5 mil na distribuição, 22,5 mil no comércio e duas mil pessoas no apoio.

Segundo Gonzaga et al. (2001), o sucesso comercial de uma nova flor de corte depende não somente de sua qualidade estética e produção, mas também de sua vida de vaso. O manuseio pós-colheita inadequado é responsável pela perda de 20 a 30\% das flores de corte que são comercializadas (HAN, 2002).
A deterioração de produtos recém colhidos é resultado de alterações fisiológicas, perda de umidade, injúrias físicas aos tecidos e invasão de microrganismos (HARDENBURG; WATADA; WANG, 1986). As flores de corte têm uma vida muito limitada após destacadas da planta mãe, pois sobrevivem com suas próprias reservas, que são geralmente reduzidas devido às próprias características fisiológicas e morfológicas dos tecidos (HARDENBURG; WATADA; WANG, 1986).

A longevidade pós-colheita de flores de corte pode ser melhorada pelo tratamento com conservantes que mantêm a qualidade e prolongam a vida de vaso das hastes pelo fornecimento de açúcares e conservação da hidratação dos tecidos (HALEVY; MAYAK, 1979).

Segundo Noordegraaf (1999), a qualidade das flores é determinada no momento da colheita, sendo, portanto, o objetivo dos tratamentos pós-colheita, não melhorar, mas sim retardar a queda na qualidade.

O uso de solução de condicionamento ("pulsing"), antes ou depois do armazenamento, é um tratamento rápido de pré-transporte ou armazenamento que afeta a fase final da vida das flores, prolongando-a mesmo após transferência para a água ou solução de manutenção. O tratamento de "pulsing" é um tratamento que satura os tecidos, utilizando, para este fim, açúcares e outros compostos químicos, como tiosulfato de prata (HALEVY; MAYAK, 1981).

Formulações especificas de "pulsing" têm sido desenvolvidas para diferentes espécies florais, e algumas vezes, para diferentes variedades (HALEVY; BYRNE; KOFRANEK, 1978). Além da concentração, a duração do tratamento de “pulsing” também é importante para a obtenção de um efeito ótimo.

O principal constituinte das soluções de "pulsing" é a sacarose em concentrações variáveis de 1 a 20\% ou maiores. Halevy e Mayak (1981) recomendam um tempo de tratamento com soluções contendo sacarose de 12 a 24 horas para a maioria das flores cortadas. 
O balanço hídrico é um fator determinante na longevidade dos órgãos das plantas, e a deficiência de água no organismo acelera a senescência (GONZAGA et al., 2001). Altos níveis de hidratação dos tecidos são em geral associados ao aumento da vida em vaso das flores de corte, enquanto perdas de 10 a $15 \%$ de sua massa fresca podem levar à morte dos tecidos (MORAES et al., 1999). A turgescência é necessária para o desenvolvimento de botões florais e também para a continuidade da atividade metabólica da flor cortada, sobre estes aspectos a sacarose tem marcante influência, pois favorece o balanço hídrico das flores cortadas. Para Halevy (1976), os açúcares translocados acumulamse nas flores aumentando a concentração de solutos, conseqüentemente favorecendo a manutenção da turgescência das pétalas.

Entretanto, em alguns casos, a sacarose pode causar efeito adverso. Moraes et al. (1999) observaram que a sacarose de 1 a $2 \%$ na solução de vaso é responsável pela plasmólise e morte do tecido de folhas de rosas de corte.

Flores de cravos, crisântemos e rosas, quando prétratadas com "pulsing" antes do armazenamento, apresentaram uma superioridade comercial quando comparadas às flores não tratadas (HALEVY; BYRNE; KOFRANEK, 1978). Moraes et al. (1997) prolongou a vida de Liatris spicata utilizando sacarose na concentração de $10 \%$ na forma de "pulsing" por 20 horas. Gonzaga et al. (2001) observaram aumento na durabilidade de cravos quando mantidos em soluções contendo sacarose a 4\% e germicida. Dias-Tagliacoso e Castro (2001) aumentaram a longevidade de hastes florais de Phalaenopsis amabilis Lindl. com a utilização de sacarose a $2 \%$ e 200ppm de ácido cítrico durante 24 horas. Stancato e Cesarino (2000) prolongaram a vida de flores cortadas de Laelia purpurata com a adição de $1 \%$ de sacarose na solução preservativa.

O objetivo deste trabalho foi avaliar a longevidade de flores de Oncidium varicosum após o acondicionamento em soluções conservantes com diferentes concentrações de sacarose.

\section{Material e Métodos}

As hastes de Oncidium varicosum, produzidas em Holambra (SP), foram transportadas para Londrina (PR) em caminhão climatizado a $5-7^{\circ} \mathrm{C}$. Foram selecionadas hastes com características semelhantes (estágio de maturação, número de flores e botões). As hastes foram cortadas em bisel sob água corrente, padronizando seu comprimento em aproximadamente $45 \mathrm{~cm}$.

Foram avaliadas a utilização de pré-tratamento com "pulsing" (15\% de sacarose por 24 horas) seguido de acondicionamento em solução de manutenção (1, 2, 3 e 4\% de sacarose) e o acondicionamento em solução de manutenção sem pré-tratamento.

Os tratamentos avaliados foram: T0 $(0 \%$ de sacarose, testemunha), T1 (1\% de sacarose), T2 (2\% de sacarose), T3 (3\% de sacarose), T4 (4\% de sacarose), T5 ( $0 \%$ de sacarose + "pulsing" de 24 horas em $15 \%$ de sacarose), T6 (1\% de sacarose + "pulsing" de 24 horas em 15\% de sacarose), T7 ( $2 \%$ de sacarose + "pulsing" de 24 horas em $15 \%$ de sacarose), T8 (3\% de sacarose + "pulsing" de 24 horas em $15 \%$ de sacarose), T9 ( $4 \%$ de sacarose + "pulsing" de 24 horas em 15\% de sacarose). Em todas as soluções foram adicionados $0,5 \mathrm{ml}$ de hipoclorito de sódio para efeito germicida.

As hastes florais que sofreram o pré-tratamento com "pulsing" (15\% de sacarose) foram acondicionadas em um recipiente contendo 2 litros da solução. Após 24 horas foram transferidas uma a uma para recipientes contendo $200 \mathrm{ml}$ da solução de manutenção. As hastes que não sofreram o prétratamento com "pulsing" foram colocadas diretamente nas soluções de manutenção. As soluções foram renovadas a cada dois dias. A temperatura foi mantida em média $25^{\circ} \mathrm{C} \pm 2^{\circ} \mathrm{C}$.

As hastes foram distribuídas em delineamento inteiramente casualizado, com dez tratamentos e cinco repetições, sendo cada haste considerada uma repetição. 
As avaliações das flores foram diárias, sob critério de notas definido para comparar e verificar a conservação dos aspectos visuais (Figura 1), sendo: Nota 1 (túrgida), Nota 2 (túrgida e pequenas manchas marrom), Nota 3 (túrgidas e sem manchas), Nota 4 (dobramento das bordas e início do fechamento), Nota 5 (flor fechada), Nota 6 (escurecimento e murcha), Nota 7 (queda da flor).

Notas superiores a três representam flores sem valor comercial, entretanto as hastes possuem flores em vários estágios de maturação (de botões a flores abertas). Neste trabalho foram consideradas apenas as flores que estavam desabrochando no início do experimento.

\section{Resultados e Discussão}

As médias das notas obtidas pelas flores sob os diferentes tratamentos pós-colheita são mostradas na Tabela 1.

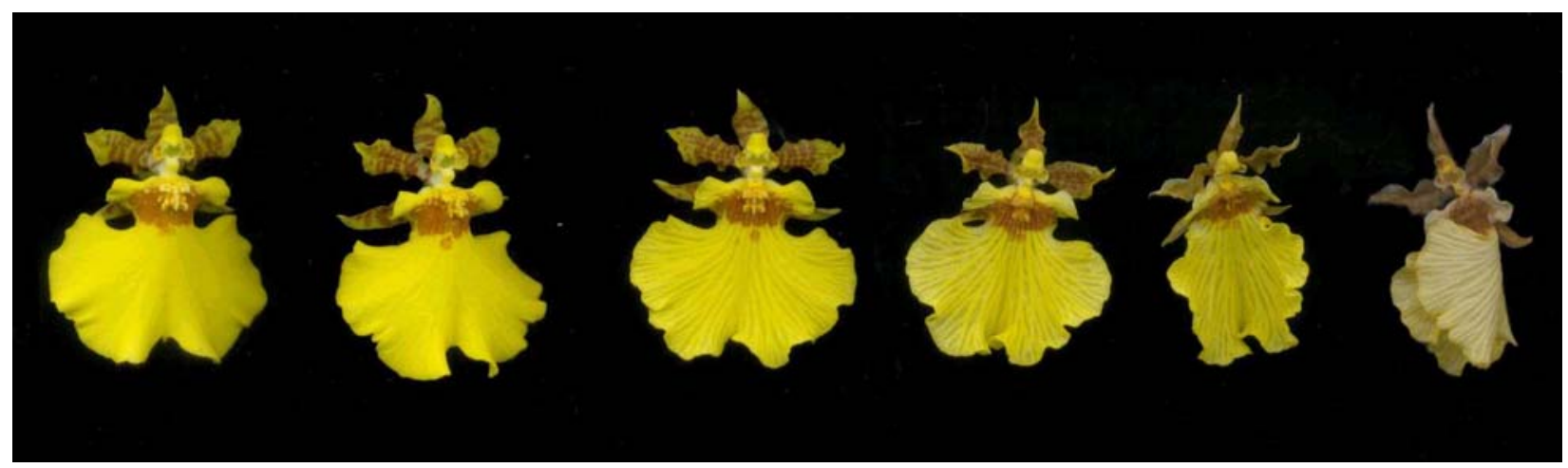

Figura 1. Critério de notas utilizadas para avaliação da qualidade estética das flores de Oncidium varicosum (da esquerda para direita 1-2-3-4-5-6).

Tabela 1.

\begin{tabular}{|c|c|c|c|c|c|c|c|c|c|c|c|c|c|c|}
\hline \multirow[t]{2}{*}{ Tratamentos } & \multicolumn{14}{|c|}{ Dias } \\
\hline & 1 & 2 & 3 & 4 & 5 & 6 & 7 & 8 & 9 & 10 & 11 & 12 & 13 & 14 \\
\hline T0 (testemunha) & 1,00 & $1,36 \mathrm{c}^{* *}$ & $2,24 \mathrm{a}$ & $3,28 \mathrm{a}$ & $4,44 a b c$ & $5,60 \mathrm{abc}$ & $6,08 \mathrm{a}$ & $7,00 \mathrm{a}$ & $7,00 \mathrm{a}$ & $7,00 \mathrm{a}$ & $7,00 \mathrm{a}$ & $7,00 \mathrm{a}$ & $7,00 \mathrm{a}$ & $7,00 \mathrm{a}$ \\
\hline $\mathrm{T} 1(1 \% *)$ & 1,00 & $1,72 \mathrm{abc}$ & $2,68 \mathrm{a}$ & $3,92 \mathrm{a}$ & $4,20 \mathrm{abc}$ & $4,72 \mathrm{bc}$ & $5,80 \mathrm{abc}$ & $7,00 \mathrm{a}$ & $7,00 \mathrm{a}$ & $7,00 \mathrm{a}$ & $7,00 \mathrm{a}$ & $7,00 \mathrm{a}$ & $7,00 \mathrm{a}$ & $7,00 \mathrm{a}$ \\
\hline T2 $(2 \%)$ & 1,00 & $1,40 \mathrm{bc}$ & $2,28 \mathrm{a}$ & $3,36 \mathrm{a}$ & $3,44 \mathrm{bc}$ & $3,72 \mathrm{c}$ & $4,92 \mathrm{c}$ & $6,44 \mathrm{bc}$ & $6,60 \mathrm{bc}$ & $6,76 \mathrm{a}$ & $6,96 \mathrm{a}$ & $7,00 \mathrm{a}$ & $7,00 \mathrm{a}$ & $7,00 \mathrm{a}$ \\
\hline T3 (3\%) & 1,00 & $1,16 \mathrm{c}$ & $2,08 \mathrm{a}$ & $3,16 \mathrm{a}$ & $3,18 \mathrm{c}$ & $3,84 \mathrm{bc}$ & $4,48 \mathrm{bc}$ & $5,52 \mathrm{bc}$ & $5,64 \mathrm{bc}$ & $6,00 \mathrm{a}$ & $6,44 \mathrm{a}$ & $6,68 \mathrm{a}$ & $6,88 \mathrm{a}$ & $7,00 \mathrm{a}$ \\
\hline T4 (4\%) & 1,00 & $1,68 \mathrm{abc}$ & $2,88 \mathrm{a}$ & $4,04 \mathrm{a}$ & $4,48 \mathrm{ab}$ & $5,60 \mathrm{abc}$ & $5,96 \mathrm{abc}$ & $6,48 \mathrm{c}$ & $6,68 \mathrm{bc}$ & $6,84 \mathrm{a}$ & $7,00 \mathrm{a}$ & $7,00 \mathrm{a}$ & $7,00 \mathrm{a}$ & $7,00 \mathrm{a}$ \\
\hline T5 ( $0 \%+$ "pulsing") & 1,00 & $1,84 \mathrm{ab}$ & $2,96 \mathrm{a}$ & $4,40 \mathrm{a}$ & $4,52 \mathrm{a}$ & $5,32 \mathrm{a}$ & $5,44 \mathrm{ab}$ & $6,00 \mathrm{c}$ & $6,24 \mathrm{c}$ & $6,60 \mathrm{a}$ & $6,64 \mathrm{a}$ & $6,80 \mathrm{a}$ & $6,96 \mathrm{a}$ & $7,00 \mathrm{a}$ \\
\hline T6 (1\%+ "pulsing") & 1,00 & $1,84 \mathrm{ab}$ & $2,80 \mathrm{a}$ & $4,20 \mathrm{a}$ & $4,72 \mathrm{ab}$ & $5,68 \mathrm{a}$ & $5,80 \mathrm{abc}$ & $6,60 \mathrm{bc}$ & $6,88 \mathrm{abc}$ & $6,96 a$ & $7,00 \mathrm{a}$ & $7,00 \mathrm{a}$ & $7,00 \mathrm{a}$ & $7,00 \mathrm{a}$ \\
\hline T7 (2\%+ "pulsing") & 1,00 & $2,04 \mathrm{a}$ & $3,12 \mathrm{a}$ & $4,76 \mathrm{a}$ & $5,04 \mathrm{a}$ & $5,64 a b c$ & $5,96 \mathrm{a}$ & $6,84 \mathrm{ab}$ & $6,92 \mathrm{ab}$ & $7,00 \mathrm{a}$ & $7,00 \mathrm{a}$ & $7,00 \mathrm{a}$ & $7,00 \mathrm{a}$ & $7,00 \mathrm{a}$ \\
\hline T8 (3\%+ "pulsing") & 1,00 & $1,72 \mathrm{abc}$ & $2,84 \mathrm{a}$ & $4,20 \mathrm{a}$ & $4,48 \mathrm{ab}$ & $5,60 \mathrm{a}$ & $5,92 \mathrm{a}$ & $6,68 \mathrm{abc}$ & $6,84 \mathrm{abc}$ & $6,96 \mathrm{a}$ & $7,00 \mathrm{a}$ & $7,00 \mathrm{a}$ & $7,00 \mathrm{a}$ & $7,00 \mathrm{a}$ \\
\hline T9 (4\%+ "pulsing") & 1,00 & $2,00 \mathrm{ab}$ & $3,08 \mathrm{a}$ & $4,60 \mathrm{a}$ & $4,92 \mathrm{a}$ & $5,88 \mathrm{a}$ & $6,00 \mathrm{a}$ & $6,40 \mathrm{bc}$ & $6,68 \mathrm{bc}$ & $7,00 \mathrm{a}$ & $7,00 \mathrm{a}$ & $7,00 \mathrm{a}$ & $7,00 \mathrm{a}$ & $7,00 \mathrm{a}$ \\
\hline
\end{tabular}

As médias das notas obtidas pelas flores foram submetidas ao Teste de Tukey (5\%) (GOMES, 1990). 
Observou-se já no segundo dia diferenças significativas entre os tratamentos. Com exceção de T8, os tratamentos que incluíram "pulsing" com 15\% de sacarose por 24 horas no início do experimento (T5, T6, T7 e T9) foram significativamente inferiores à testemunha (T0) e ao T3 (3\% de sacarose na solução de manutenção). Este resultado pode ser devido à sensibilidade das hastes de Oncidium varicosum a concentrações maiores que $4 \%$ na solução de manutenção e a concentrações tão altas quanto a que foi usada no pré-tratamento com “pulsing". Segundo Han (2002), Zinnias e Heuchera sofrem danos quando tratadas com concentrações de sacarose maiores que $1 \%$. Dias-Tagliacoso e Castro (2001) aumentaram a longevidade de hastes florais de Phalaenopsis amabilis Lindl. com a utilização de sacarose a $\%$ e 200ppm de ácido cítrico durante 24 horas. Stancato e Cesarino (2000) prolongaram a vida de flores cortadas de Laelia purpurata com a adição de $1 \%$ de sacarose na solução preservativa.

No terceiro e quarto dia, não ocorreram diferenças significativas entre os tratamentos, sendo que no terceiro dia os tratamentos 7 e 9 já apresentavam flores sem valor comercial e os tratamentos 4, 5, 6 e 8 apresentavam valores bem próximos da perda de valor comercial. Entretanto, no quarto dia, todos os tratamentos obtiveram médias superiores a três, perdendo valor comercial. Como não houveram diferenças significativas entre os tratamentos neste dia, nenhum dos tratamentos demonstrou superioridade na manutenção das características das flores.

No quinto dia o tratamento T3 apresentou resultado superior a todos os tratamentos com "pulsing" (T5, T6, T7, T8, T9) e ao T4 (4\% de sacarose na solução de manutenção), mas continuou não sendo estatisticamente superior à testemunha.

Os resultados do sexto dia mostram que T1, T2 e T3 (1, 2 e 3\% de sacarose na solução de manutenção, respectivamente) foram superiores a todos os tratamentos com "pulsing” com exceção de T7 (2\% de sacarose na solução de manutenção + "pulsing"). Novamente nenhum tratamento foi significativamente superior à testemunha.

No sétimo dia o tratamento T2 (2\% de sacarose na solução de manutenção) foi superior aos tratamentos T5, T7, T8 e T9 (0, 2, 3 e 4\% de sacarose na solução de manutenção, respectivamente, + "pulsing"). Os tratamentos T2 e T3 apresentaram melhores resultados que a testemunha (Tabela 1).

Os dados do oitavo dia mostram que os tratamentos $\mathrm{T} 0$ e $\mathrm{T} 1$ foram inferiores a quase todos os outros tratamentos, com exceção de T7 e T8. No nono dia os tratamentos T0 e T1 foram inferiores aos tratamentos T2, T3, T4 e T5, porém as flores já apresentavam nota superior a 5 , ou seja, sem valor comercial.

Do dia dez ao quatorze, não houve diferenças significativas entre os tratamentos.

Os tratamentos T1, T2 e T3 (1, 2 e 3\% na solução de manutenção, respectivamente, sem "pulsing") foram eficientes em promover abertura de botões florais e manter a haste viável comercialmente por mais tempo. Segundo Wilkins (2002), após colhidas, a maioria das flores precisam ser acondicionadas em soluções promotoras de abertura de botões. Estas soluções geralmente contêm germicida e açúcar, a mesma composição das soluções avaliadas neste experimento.

O tratamento com estas soluções promotoras de abertura de botões deve ser realizado em temperaturas ambientes entre $21-27^{\circ} \mathrm{C}$ (WILKINS, 2002). O experimento foi realizado em temperaturas próximas a este intervalo $\left(25^{\circ} \mathrm{C} \pm 2^{\circ} \mathrm{C}\right)$, mostrando que os tratamentos $\mathrm{T} 1, \mathrm{~T} 2$ e $\mathrm{T} 3$ podem ser estudados como potenciais soluções promotoras de abertura de botões de Oncidium varicosum. As principais vantagens de se promover a abertura de botões após o corte são a redução no tempo de cultivo, aumento na densidade dos pacotes, diminuição dos danos mecânicos, diminuição da área superficial e redução da perda de água. 
A concentração ótima de açúcar usada em tratamentos pós-colheita varia significativamente dependendo da flor a ser tratada. A maioria das flores se beneficia com $2 \%$ de açúcar nas soluções de manutenção (Han, 2002). Nos tratamentos com "pulsing" a concentração de açúcar pode chegar a mais de 20\% (HALEVY; MAYAK, 1981). Entretanto, em alguns casos, a sacarose pode causar efeito adverso (HAN, 2002). Moraes et al. (1999) observaram que a sacarose de 1 a $2 \%$ na solução de vaso é responsável pela plasmólise e morte do tecido de folhas de rosas de corte.

A temperatura ambiente durante o experimento $\left(25^{\circ} \mathrm{C} \pm 2^{\circ} \mathrm{C}\right)$ estava acima da encontrada em floriculturas, que geralmente possuem condicionadores de ar $\left(20^{\circ} \mathrm{C}\right)$, entretanto representa a condição encontrada no local de consumo, geralmente sem refrigeração. A boa aceitação de uma flor de corte depende também da sua durabilidade nas condições de consumo.

Segundo McConchie (2002) a temperatura é determinante para a taxa de respiração, quanto mais alta a temperatura, mais alta a taxa de respiração e mais rápido a flor irá morrer. Altas temperaturas aumentam a perda de água e aceleram o envelhecimento das plantas e flores (MORAES et al., 1999).

\section{Conclusões}

Nas condições estudadas nenhum dos tratamentos avaliados mostrou-se eficiente no aumento da longevidade de flores de Oncidium varicosum após a colheita, que foi de três a quatro dias.

\section{Referências}

DiAS-TAGLiAcoso, G. M.; CASTRO, C. E. F. Prolongamento da longevidade pós-colheita de falenopse. In: CONGRESSO BRASILEIRO DE FLORICULTURA E PLANTAS ORNAMENTAIS, 13, 2001. São Paulo. Resumos... São Paulo: Sociedade Brasileira de Floricultura e Plantas Ornamentais, 2001. p.27.

GOMES, F. P. Testes não paramétricos. In:___ Curso de estatística experimental. 13 ed. Piracicaba: Livraria Nobel, 1990. p. 383-401

GONZAGA, A. R.; MOREIRA, L. A.; LONARDONI, F.; FARIA, R. T. Longevidade pós-colheita de inflorescências de girassol afetada por nitrato de prata e sacarose. Revista Brasileira de Horticultura Ornamental, Campinas, v.7, n.1, p.73-77, 2001.

HALEVY, A. H. Treatments to improve water balance of cut flowers. Acta Horticulturae, Sweden, v.64, p. 223-230, 1976.

HALEVY, A. H.; BYRNE, G. T.; KOFRANEK, M. A. Evolution of postharvest handling methods for transcontinental truck shipments of cut carnations, crysanthemum and roses. Journal of the American Society for Horticultural Science, Alexandria, v.103, n.2, p. 151$155,1978$.

HALEVY, A. H.; MAYAK, S. Transport and conditioning of cut flowers. Acta Horticulturae, Sweden, v.43, p.291306, 1978.

Senescence and postharvest physiology of cut flowers - Part 1. Horticultural Reviews, Westport, v.1, p.204-236, 1979.

Senescence and postharvest physiology of cut flowers - Part 2. Horticultural Reviews, Westport, v.1, p.59-143, 1981.

HAN, S. S. Sugar and acidity in preservative solutions for field-grown cut flowers. Disponível em: <http:// www.umass.edu/umext/programs/agro/floriculture/ floral_facts/presvcut.html>. Acesso em: 20 dez. 2002.

HARDENBURG, R. E., WATADA, A. E.; WANG, C. Y. The commercial storage of fruits, vegetables, florists and nursery stocks. Washington: USDA, 1986. 
INSTITUTO BRASILEIRO DE FLORICULTURA. Informativo Ibraflor. Disponível em: <http:// www.ibraflor.com.br/ibraflor/index.php?id=23>. Acesso em: 14 jun. 2005.

McCONCHIE, R. Postharvest care of cut flowers. Disponível em: <http:/www.sydneyflower.com.au/Flower/ lores/postharvest.htm>. Acesso em: 20 dez. 2002.

MORAES, P. J.; FINGER, F. L.; BARBOSA, J. G.; SILVA, D. J. H. Efeito do "pulsing" com sacarose sobre o índice de sobrevivência de Chysanthemum leucanthemum L. Revista Brasileira de Horticultura Ornamental, Campinas, v.3, n.2, p.80-84, 1997.

MORAES, P.J.; CECON, P.R. FINGER, F.L.;BARBOSA, J.G.; ALVARES, V.S. Efeito da refrigeração e do condicionamento em sacarose sobre a longevidade de inflorescências de Strelitzia reginae Ait. Revista Brasileira de Horticultura Ornamental, Campinas, v.5,n.2,p.151-156, 1999.
NOORDEGRAAF, C. Problems of postharvest management in cut flowers. Acta Horticulturae, 1999. Disponível em: $<$ http://www.actahort.org/books/482/482_6.htm>. Acesso em: 20 dez. 2002.

STANCATO, G. C.; CESARINO, F. Longevidade e durabilidade de flores de Laelia purpurata Lindl. (Orchidaceae). Revista Brasileira de Horticultura Ornamental, Campinas, v.6, n.1/2, p.69-74, 2000.

WILKINS, H. F. Postharvest care of cut flowers. Disponível em: <http://www.aginfonet.com/agricarta/ content/sk_driedflower/postharvest_care.html $>$. Acesso em: 20 dez. 2002. 